\title{
Commentary Pregnancy-induced changes in cell-fate in the mammary gland
} Melanie R Ginger and Jeffrey M Rosen

\author{
Department of Molecular and Cellular Biology, Baylor College of Medicine, Houston, Texas, USA \\ Corresponding author: Melanie R Ginger (e-mail: mginger@bcm.tmc.edu) \\ Published: 25 April 2003 \\ Breast Cancer Res 2003, 5:192-197 (DOI 10.1186/bcr603) \\ (C) 2003 BioMed Central Ltd (Print ISSN 1465-5411; Online ISSN 1465-542X)
}

\begin{abstract}
The protective effect of an early full-term pregnancy is a well established phenomenon; in contrast, the molecular and cell-specific mechanisms that govern parity-specific changes in the mammary gland have not been well described. Recent studies signify a dramatic advance in our understanding of this phenomenon, and indicate a 'cell fate' model for parity-related changes that lead to protection against breast cancer.
\end{abstract}

Keywords: breast cancer, cell fate, epigenetic, mechanism, pregnancy, protection

\section{Introduction}

On the basis of epidemiologic studies and pathology, breast cancer appears to be an extremely complex, multifactorial disease. In spite of our growing understanding of the molecular aberrations that lead to cancer, and the role of endocrine signaling pathways in the progression of the disease, there are few common links pointing to an individual's susceptibility to breast cancer. However, one consistent finding is the correlation between the timing of normal endocrine-related developmental events and breast cancer risk [1]. In particular, there is strong epidemiologic evidence that women who experience a full-term pregnancy early in their lives have a significantly reduced risk for developing breast cancer [2-5]. This has been corroborated by numerous experimental studies in rodents that resoundingly demonstrated the protective effect of an early full-term pregnancy against mammary tumors (reviewed in [6]). In addition, hormonal mimicry, by treatment with estrogen and progesterone $(E+P)$ or human chorionic gonadotropin for a period of at least 21 days, can be equally effective for inducing a refractory state that is similar to that afforded by an early full-term pregnancy [6-13].

The observation that the protective effect of an early fullterm pregnancy can be accurately reproduced in rodents has lead to the development of defined animal models for studying this parity-related phenomenon. For many years, tumor-susceptible rat models were the mainstay of this experimental effort; however, there is a growing body of evidence suggesting that several strains of mice may also be appropriate models for these studies. Despite the wealth of literature supporting the role of endocrine-mediated processes in parity-related refractoriness, little is known of the molecular mechanisms that govern pregnancy-specific developmental changes in the mammary gland. In this commentary, we consider the contributions made by several recent publications to the advancement of our understanding of the molecular and cell-specific changes apparent in the parous (protected) gland. In this context, 'persistent changes' are defined as alterations that occur as a result of parity but that endure far beyond the initial stimulatory event. Taken together, these findings provide important insights into the strategies that might be employed for future studies of this parity-related phenomenon.

\section{A global profile for molecular changes in the parous mammary gland}

As a preliminary step in elucidating the molecular mechanisms that underlie parity-related protection, we and others have searched for molecular changes in the parous mammary gland. In the first study of this kind [14], we employed the Wistar-Furth rat model in conjunction with 
suppression subtractive hybridization to isolate molecular biomarkers for the protective effect of an early full-term pregnancy. Using this approach, we identified a set of 100 independent (nonredundant) markers that were persistently upregulated in the glands of 21 -day $\mathrm{E}+\mathrm{P}$ treated $(20 \mu \mathrm{g}$ estrogen/20 mg progesterone), 28-day involuted rats, as compared with age-matched virgin (AMV) control animals.

In an elaborate and rigorously executed study, D'Cruz and coworkers [15] applied global profiling metholodologies to examine persistent changes in the pattern of gene expression, as a function of parity, in the rodent mammary gland. Using oligonucleotide arrays, they performed a quantitative assessment of the expression levels of 5500 genes in the mammary glands of parous and nonparous FVB mice. In addition, they verified the expression of a subset of candidate markers by Northern analysis.

One concern raised by this type of analysis is that the findings may not be applicable across different species or even across different strains of mice [6]. Furthermore, although the protective effect of parity has been well characterized in many rat models of chemical carcinogenesis, these studies have only been performed in three strains of mouse $[16,17]$. To corroborate their findings, D'Cruz and coworkers examined the expression profile of a smaller subset of these markers as a function of parity in the mammary glands of $129 \mathrm{SvEv}$ and Balb/c mice, as well as Lewis and Sprague-Dawley rats. As a result of this analysis, they identified a subset of $\mathbf{3 8}$ genes that exhibited persistent alterations in their pattern of expression in parous versus nonparous rodents.

\section{Global profiling: a predictive tool for parity- specific change in mammary gland}

To extend their findings, D'Cruz and coworkers [15] tested whether the expression pattern of this subset of markers was sufficient to predict, in a blinded manner, the reproductive history of a sample. To examine the predictive merit of their approach, they performed microarray analysis with an independent group of mammary gland RNA samples and then used hierarchical clustering, based on the expression profiles of their 38 genes, to determine the reproductive history of the tissues of origin. These results elegantly demonstrate that this data set can be effectively and reproducibly used as a predictive indicator of parity-specific protection; such findings raise the prospect of developing a prognostic tool that is also translational to human studies.

\section{Parity results in changes to growth-regulatory pathways in rodent mammary gland}

D'Cruz and coworkers [15] showed that genes that encode growth factors such as amphiregulin (A-reg), pleiotrophin (Ptn), leptin $(O b)$ and insulin-like growth factor-1 (Igf-1), which may potentially be involved in epithelial proliferation, are downregulated in the parous involuted gland. In the case of Ptn and A-reg, at least, downregulation is observed as early as mid-pregnancy (day 12), and these genes remain persistently downregulated following 21 days of lactation and 28 days of postlactational involution. Conversely, genes that encode markers of the transforming growth factor (TGF)- $\beta_{3}$ signaling pathway are persistently upregulated following parity and remain elevated (as compared with AMV control animals) for up to 30 weeks following postlactational involution. Previous studies have shown that TGF- $\beta_{3}$ mRNA is markedly upregulated during involution, reaching peak expression during the second phase of this process [18]. Therefore, the persistent expression of TGF- $\beta_{3}$ observed by D'Cruz and coworkers could be a function of involution rather than parity. From our own studies (Ginger M, Rosen J, unpublished observations) we have also observed the downregulation of certain growth-promoting molecules; in contrast, markers involved in cell cycle control (Cdc42, Rb) or the modulation of the TGF- $\beta$ signaling pathway (Fstl-1, Nrln-1) are upregulated in the parous mammary gland [14]. Interestingly, the observed changes in the level of insulin-like growth factor-1 mRNA may reflect an alteration in systemic growth hormone levels reported previously [10]. Altogether, these findings tend to support the notion that there are persistent changes in growth regulatory pathways in the parous mammary gland.

The downregulation of mRNA for certain growth-stimulatory molecules is a tantalizing observation, in particular Ptn, Igf-1 and A-reg, because over-expression of these growth factors has been associated with human breast cancer. However, these findings alone do not provide sufficient explanation for the refractory nature of the parous gland because it has been adequately demonstrated that the gland can re-enter the proliferative phase during subsequent pregnancies. However, as suggested by D'Cruz and coworkers [15] and previously by Sivaraman and colleagues [17], the role of these molecules may only become evident once the gland is exposed to further proliferative stimuli. Moreover, it is possible that the downregulation of growth-promoting molecules and the upregulation of the TGF- $\beta_{3}$ signaling pathway may work in concert with one another, as well as other factors, to confer an altered response to subsequent proliferative stimuli.

\section{p53 expression is correlated with a proliferative block}

Previously, Sivaraman and coworkers [9] showed that one of the earliest consequences of carcinogen exposure is a proliferative burst in the epithelial compartment of the nonparous gland. In contrast, proliferation is abrogated in glands of parous or E+P-treated rats, although the pathways that govern this differential proliferative response have not yet been elucidated. In their recent publication, a potential mechanism for this proliferative block is explored. 
Using the Wistar-Furth rat model described in their previous study [9], those investigators examined the expression pattern of p53 protein in response to hormonal stimulation and exposure to the chemical carcinogen methylnitroso urea (MNU) [17]. Rats aged 45 days were subjected to a priming dose of estradiol benzoate (to synchronize estrus) and then treated with $\mathrm{E}+\mathrm{P}(20 \mu \mathrm{g}$ estrogen $/ 20 \mathrm{mg}$ progesterone, subcutaneously) for a period of 21 days. This represents a modest dose of hormones, but it is sufficient to confer $82 \%$ protection against MNU-induced tumorigenesis. Treatment with $\mathrm{E}+\mathrm{P}$ induced the upregulation and nuclear sequestration of p53 protein; p53 was up-regulated as early as 3 days after $E+P$ treatment and remained elevated for the remainder of the 21-day treatment period. Strikingly, these levels were persistently elevated 28 days after hormone withdrawal, as compared with nonparous AMV, and remained elevated for at least 3 days following exposure to MNU. Pregnancy induced a similar pattern of expression; p53 was upregulated during pregnancy, remained persistently elevated after a 28-day involution period, and was localized to the nucleus of mammary epithelial cells. Nuclear accumulation of p53 preceded the proliferative block described previously and resulted in transcriptional activation of p53 target genes (MDM2 and p21 ${ }^{\mathrm{CIP} 1 / \mathrm{WAF} 1)}$ at 3 days post-MNU exposure.

To evaluate the mouse as a suitable model for these parity-related changes, Sivaraman and coworkers [17] repeated their experiments using BALB/c mice, and were able to recapitulate their findings from the E+P-treated Wistar-Furth rat. Once again, nuclear accumulation of p53 and induction of p21 preceded the proliferative block following carcinogen exposure, suggesting that they may contribute mechanistically to this event. These observations are strengthened by the finding that p53-mediated apoptosis, in response to $\gamma$-irradiation, in the pregnant and involuting gland differs significantly from that in the glands of virgin animals [19]. Given the fact that aberrations in p53 expression and/or function are often associated with breast cancer, parity-specific changes in p53 expression are likely to play an important role in protection against tumorigenesis.

\section{Pregnancy results in changes in the cellular composition of the mammary gland}

As a consequence of their profiling studies, D'Cruz and coworkers [15] provided evidence to suggest that there are persistent alterations in the cellular composition of the parous mammary gland: the persistent presence of hematopoietic cells, or cells with heomatopoietic-like properties, as well as changes in cytokine signaling pathways within the epithelium. At this point it is not clear whether the presence of cells with hematopoietic-like properties is due to the existence of lymphocytes and macrophages within the gland, or to the recruitment of certain epithelial
By independent means, Wagner and coworkers [20] also demonstrated alterations in the epithelial population of the parous mammary gland. Using double transgenic mice, expressing Cre-recombinase under the control of the whey acidic protein (WAP) gene promoter and carrying a floxed-Stop-lacZ knock-in cassette (Rosa-lox-Stop-loxlacZ; hereafter referred to as Rosa-LacZ), those investigators were able to track the fate of WAP-Cre-expressing mammary epithelial cells during pregnancy and involution. The elegance of this approach derives from the ability of the Cre-recombinase to 'inscribe' its effect (i.e. recombination between loxP sequences), leading to permanent activation of the lacZ reporter gene in cells in which WAPCre expression has occurred. Thus, any cell that responds to the appropriate hormonal and developmental signal to express WAP-Cre retains a lasting signature ( $\beta$-galactosidase expression) of this developmentally specific event.

Because expression of the WAP-Cre transgene closely mirrors that of the endogenous WAP gene, hormonal and developmental changes typical of mid to late pregnancy are required for its expression. During involution the intricate, differentiated alveolar structure of the epithelium diminishes through a combination of apoptotic and remodeling processes, and the gland regresses to a more quiescent state that is similar to that of the mature virgin gland. WAP gene, and therefore presumably WAP-Cre, expression ceases during the first few days of involution. However as Wagner and coworkers [20] demonstrated, a specific component of the epithelium retains $\beta$-galactosidase expression 3-4 weeks after postlactational involution.

Thus, they identified a novel epithelial cell population originating from a parity-specific event, but which is distinct from the majority of the cells in the pregnant mammary gland. The ability of these cells not only to differentiate but also to survive involution distinguishes them as a unique subpopulation of the mammary epithelium. Furthermore, this observation implies that differentiation alone is not the basis of their unique properties, but that some other factor is responsible for their enduring presence in the parous mammary gland.

Strikingly, these $\beta$-galactosidase-expressing cells are confined primarily to the regressed terminal ductal lobular-like structures (TDLUs in humans) at the extremity of the ductal tree. As we have previously shown, two markers of parity-related protection, RbAp46 (a component of the Sin3A/HDAC, Mi-2/NuRD and histone methyltransferase complexes) and G.B7 (a noncoding RNA), are likewise expressed in a localized manner in the TDLU-like structures of the involuted mammary gland [14].

\section{A unifying hypothesis}

Taken together, these findings suggest that there are permanent alterations in the epithelial compartment of the 
(a)



(b) Epigenetic mechanisms governing changes in cell fate

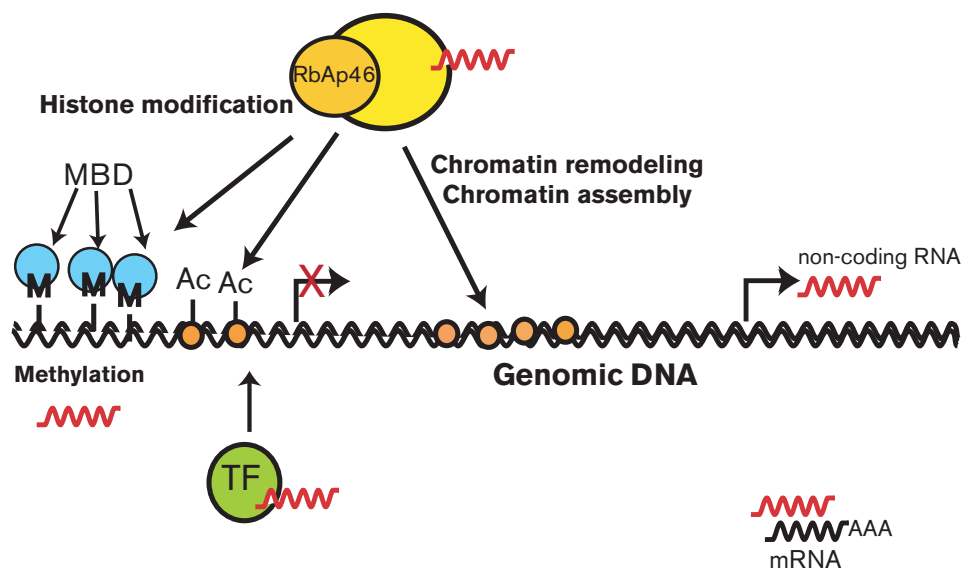

Unified model for parity-specific changes in the mammary gland. (a) Summary of reported parity-dependent changes in the mammary gland. Pregnancy induces multiple changes in the mammary epithelial cells, including nuclear accumulation of p53 and induction of whey acidic protein (WAP)-Cre expression (and subsequently $\beta$-galactosidase expression, indicated by blue cells) in WAP-Cre/Rosa-LacZ mice, and alteration in gene activity perhaps leading to changes in cell fate. During involution, a large component of the epithelium is eliminated through programmed cell death; however, a specific subpopulation is able to circumvent this process. The involuted mammary gland is characterized by persistent changes in gene expression, nuclear localization of p53, and an altered proliferative capacity in response to carcinogen. (b) Potential mechanism for the parityspecific changes in cell fate: Pregnancy invokes epigenetic changes affecting cell fate in the parous mammary gland. Epigenetic changes may be induced by a number of mechanisms, including chromatin remodeling, DNA methylation/demethylation, and histone modification, and as a result of de novo chromatin assembly. RbAp46 is a component of a number of complexes responsible for these processes, including the NuRD complex [23], which also contains methyl-CpG binding domain proteins (MBD). The common presence of RbAp46 in these complexes might thus provide a mechanism for sequential shuttling between these different functions, and in addition provides a link between DNA methylation and chromatin regulation. Noncoding RNAs have also been implicated in these changes in gene expression, through their association with chromatin remodeling, histone acetylation/deacetylation, and transcription factor complexes, as well as RNA interference [24,25]. Epigenetic changes can regulate cell fate in a number of ways, for instance by altering gene activity, by providing a signal for survival or proliferation, and by mediating responses to DNA damage. In addition, it is likely that there is continual cross-talk between the epithelium and the stroma, thus providing an additional level of epigenetic regulation in the parous mammary gland. Ac, acetylated histones; M, methylated cytosine; TF, transcription factor. 
parous mammary gland (Fig. 1a). These changes include, but are not limited to, the following: an alteration in the signaling pathways governing cell-growth (and possibly also chemoattractant properties or the expression of hematopoietic markers); the ability to differentiate, survive apoptosis, and still retain the propensity for self-renewal; and the ability to circumvent proliferative stimuli, possibly through the transcriptional activation of p53 target genes. These changes endure beyond the primary phase of stimulation due to the hormonal manipulation or pregnancy, persisting 1 or even 6 months after this initial inductive event. In addition, changes in systemic hormone levels, as well as in the mammary stroma [21], may also participate in the protective effects of an early pregnancy.

How does pregnancy impose such persistent changes on the mammary gland? Wagner and coworkers [20] suggested that pregnancy confers a 'functional memory' on the mammary epithelium. Sivaraman and colleagues [17] hypothesized that pregnancy results in changes to the 'developmental fate' of a subpopulation of epithelial cells. Together, these studies suggest that pregnancy alters the fate of a specific population of epithelial cells, but how is cell fate altered by pregnancy? We propose that epigenetic factors might provide a unifying mechanism for this process (Fig. 1b) [14]. Epigenetic factors tell a cell who it is, where it resides, and what type of function it can perform. Changes in epigenetic regulation (through the processes of DNA methylation, histone modification, and chromatin remodeling) frequently accompany developmental processes, and thus provide a lasting signal that restricts the pattern of gene expression in those cells, long after the inductive event has been removed [22]. Such changes could thus provide an enduring 'memory' that determines cell fate and prevents cell-lineage aberrations that lead to cancer.

\section{Conclusion}

Taken in concert, these studies, as well as our own findings, provide several important conclusions. First, parity results in persistent changes in the pattern of gene expression in the rodent mammary gland. Second, the molecular changes induced by $\mathrm{E}+\mathrm{P}$ treatment (hormonal mimicry) are mirrored by similar changes in the parous gland. Third, the molecular, proliferative, and cell-specific changes observed in the parous (or E+P-treated) mouse recapitulate the parity-related alterations seen in the rat. Fourth, parity results in an altered epithelial population in the rodent mammary gland. Such conclusions provide new insights into the strategies we might use to approach this question in the future and, in particular, they point to a new experimental model that recognizes the utility of transgenic and knockout mice as a complement to the traditional rat model. Finally, the generality of this model offers the hope that we can translate these findings to the prevention of human disease, possibly by mimicking these developmental events pharmacologically for the chemoprevention of breast cancer.

\section{Competing interests}

None declared.

\section{Acknowledgments}

We wish to thank Daniel Medina and Kathryn Schwertfeger for their critical reading of the manuscript. These studies were supported by U.S. Department of Defense BRCP grant DAMD17-00-1-0137 and by $\mathrm{NIH}$ grant CA 64255

\section{References}

1. Chodosh LA, D'Cruz CM, Gardner HP, Ha SI, Marquis ST, Rajan JV, Stairs DB, Wang JY, Wang M: Mammary gland development, reproductive history, and breast cancer risk. Cancer Res 1999, 59(suppl):1765s-1771s; discussion 1771s-1772s.

2. Kelsey JL, Gammon MD: The epidemiology of breast cancer. CA Cancer J Clin 1991, 41:146-165.

3. MacMahon B, Cole P, Lin TM, Lowe CR, Mirra AP, Ravnihar B, Salber EJ, Valaoras VG, Yuasa S: Age at first birth and breast cancer risk. Bull World Health Organ 1970, 43:209-221.

4. Rosner B, Colditz GA, Willett WC: Reproductive risk factors in a prospective study of breast cancer: the Nurses' Health Study. Am J Epidemiol 1994, 139:819-835.

5. Pike MC, Krailo MD, Henderson BE, Casagrande JT, Hoel DG: 'Hormonal' risk factors, 'breast tissue age' and the age-incidence of breast cancer. Nature 1983, 303:767-770.

6. Sivaraman L, Medina D: Hormone-induced protection against breast cancer. J Mammary Gland Biol Neoplasia 2002, 7:77-92.

7. Russo $\mathrm{IH}$, Koszalka M, Russo J: Human chorionic gonadotropin and rat mammary cancer prevention. J Natl Cancer Inst 1990, 82:1286-1289.

8. Russo $\mathrm{IH}$, Koszalka M, Russo J: Effect of human chorionic gonadotropin on mammary gland differentiation and carcinogenesis. Carcinogenesis 1990, 11:1849-1855.

9. Sivaraman L, Stephens LC, Markaverich BM, Clark JA, Krnacik S, Conneely OM, O'Malley BW, Medina D: Hormone-induced refractoriness to mammary carcinogenesis in Wistar-Furth rats. Carcinogenesis 1998, 19:1573-1581.

10. Thordarson G, Jin E, Guzman RC, Swanson SM, Nandi S, Talamantes F: Refractoriness to mammary tumorigenesis in parous rats: is it caused by persistent changes in the hormonal environment or permanent biochemical alterations in the mammary epithelia? Carcinogenesis 1995, 16:2847-2853.

11. Grubbs CJ, Farnell DR, Hill DL, McDonough KC: Chemoprevention of N-nitroso-N-methylurea-induced mammary cancers by pretreatment with 17 beta-estradiol and progesterone. J Nat/ Cancer Inst 1985, 74:927-931.

12. Guzman RC, Yang J, Rajkumar L, Thordarson G, Chen X, Nandi S: Hormonal prevention of breast cancer: mimicking the protective effect of pregnancy. Proc Natl Acad Sci USA 1999, 96: 2520-2525.

13. Huggins C, Moon JRC, Morii S: Extinction of experimental mammary cancer, I. Estradiol-17b and progesterone. Proc Natl Acad Sci USA 1962, 48:379-386.

14. Ginger MR, Gonzalez-Rimbau MF, Gay JP, Rosen JM: Persistent changes in gene expression induced by estrogen and progesterone in the rat mammary gland. Mol Endocrinol 2001, 15: 1993-2009.

15. D'Cruz CM, Moody SE, Master SR, Hartman JL, Keiper EA, Imielinski MB, Cox JD, Wang JY, Ha SI, Keister BA, Chodosh LA: Persistent parity-induced changes in growth factors, TGFbeta3, and differentiation in the rodent mammary gland. $\mathrm{Mol}$ Endocrinol 2002, 16:2034-2051.

16. Medina D, Smith GH: Chemical carcinogen-induced tumorigenesis in parous, involuted mouse mammary glands. J Natl Cancer Inst 1999, 91:967-969.

17. Sivaraman L, Conneely OM, Medina D, O'Malley BW: p53 is a potential mediator of pregnancy and hormone-induced resistance to mammary carcinogenesis. Proc Natl Acad Sci USA 2001, 98:12379-12384.

18. Faure E, Heisterkamp N, Groffen J, Kaartinen V: Differential expression of TGF-beta isoforms during postlactational mammary gland involution. Cell Tissue Res 2000, 300:89-95. 
19. Minter LM, Dickinson ES, Naber SP, Jerry DJ: Epithelial cell cycling predicts p53 responsiveness to gamma-irradiation during post-natal mammary gland development. Development 2002, 129:2997-3008.

20. Wagner KU, Boulanger CA, Henry MD, Sgagias M, Hennighausen L, Smith GH: An adjunct mammary epithelial cell population in parous females: its role in functional adaptation and tissue renewal. Development 2002, 129:1377-1386.

21. Bemis LT, Schedin P: Reproductive state of rat mammary gland stroma modulates human breast cancer cell migration and invasion. Cancer Res 2000, 60:3414-3418.

22. Muller $\mathrm{C}$, Leutz $\mathrm{A}$ : Chromatin remodeling in development and differentiation. Curr Opin Genet Dev 2001, 11:167-174.

23. Wolffe AP, Urnov FD, Guschin D: Co-repressor complexes and remodelling chromatin for repression. Biochem Soc Trans 2000, 28:379-386.

24. Dernburg AF, Karpen GH: A chromosome RNAissance. Cell 2002, 111:159-162.

25. Mattick JS: Non-coding RNAs: the architects of eukaryotic complexity. EMBO Rep 2001, 2:986-991.

\section{Correspondence}

Melanie R Ginger, Department of Molecular and Cellular Biology, Baylor College of Medicine, 1 Baylor Plaza, Houston, TX 77030, USA. Tel: +1

713798 6217; fax: +1 7173798 8012; e-mail:mginger@bcm.tmc.edu 